\title{
MedienPädagogik
}

Zeitschrift für Theorie und Praxis der Medienbildung

\section{Welche professionellen Handlungskompetenzen benötigen Lehrpersonen im Kontext der Digitalisierung in der Schule?}

\author{
Theoretische Diskussion unter Berücksichtigung der Perspektive \\ Lehramtsstudierender
}

Ramona Lorenz und Manuela Endberg

\begin{abstract}
Zusammenfassung
Die gestiegene Digitalisierung in allen Lebensbereichen bringt neue Anforderungen für Schulen und insbesondere für Lehrpersonen mit sich, um die Schülerinnen und Schüler auf das Leben in der digitalisierten Welt vorzubereiten und Potenziale digitaler Medien im Unterricht lernförderlich einzubinden. Entsprechend ist ein theoretischer Rahmen erforderlich, der die professionellen Handlungskompetenzen von Lehrpersonen im Kontext der Digitalisierung umfassend beschreibt und eine Orientierung für die Lehrerbildung bietet. Der vorliegende Beitrag diskutiert in diesem Zusammenhang ausgewählte etablierte und für die internationale Anschlussfähigkeit relevante Ansätze, womit ein originärer Beitrag für die Entwicklung von Konzepten für die Lehrerbildung geleistet wird. Zudem wird der Blick auf die Perspektive von Lehramtsstudierenden gerichtet, für die solche Konzepte aktuell und zukünftig von entscheidender Relevanz sind. Mit einer qualitativ-exploratorischen Herangehensweise wird die Einschätzung von Lehramtsstudierenden hinsichtlich der Potenziale und der Praxistauglichkeit der ausgewählten Ansätze ergänzend betrachtet. Der Beitrag schliesst mit einem Fazit zu Herausforderungen für die Entwicklung eines umfassenden Konzepts professioneller Handlungskompetenzen von Lehrpersonen sowie für die verknüpfte und phasenübergreifende Integration der Digitalisierung in der Lehrerbildung.
\end{abstract}

Which professional competencies do teachers need in the context of ICT in schools? Theoretical discussion and consideration of pre-service teachers' perspectives

\begin{abstract}
The advanced digitalization in all areas of life implies new demands for schools and teachers in order to prepare students for the life in the digital age as well as to integrate ICT efficiently in instruction to promote learning processes. Thus, a theoretical framework is needed which describes the professional competencies of teachers in the context of ICT comprehensively and provides an orientation for teacher education. The paper at
\end{abstract}


hand discusses selected established theoretical approaches which are important for the compatibility in an international comparison of teacher education. Furthermore the perspective of pre-service teachers is taken into account due to the relevance of ICT-related concepts for future teacher education. With qualitative methods an exploratory approach to pre-service teachers' perceptions of potentials and suitability of the theoretical constructs is supplemented. Finally, challenges for the development of a comprehensive concept of teachers' professional competencies with regard to ICT in schools and for the implementation of ICT in all phases of teacher education are emphasized.

Konzeption professioneller Kompetenzen von Lehrpersonen im Kontext der Digitalisierung

Die Digitalisierung verändert alle beruflichen, privaten und gesellschaftlichen Lebensbereiche nachhaltig. Es steht daher ausser Frage, dass Schulen ihren Erziehungsund Bildungsauftrag auch darauf ausrichten müssen, die Schülerinnen und Schüler auf diese veränderten Lebensbedingungen vorzubereiten. Aus pädagogischer Sicht sprechen viele Vorteile für die gezielte Nutzung digitaler Medien für das fachliche und überfachliche Lernen (Cress et al. 2018; Netzwerk Digitale Bildung 2018). Dass diese allzu häufig jedoch nicht ausgeschöpft werden, liegt in hohem Masse an der fehlenden professionellen Handlungskompetenz der Lehrpersonen im Kontext der Digitalisierung. Vorwerfen lassen müssen sich die Lehrpersonen allerdings kaum etwas, denn in der Regel bestand für sie kaum Gelegenheit in der Lehramtsausbildung entsprechende Kompetenzen zu erwerben (Eickelmann et al. 2016; Schiefner-Rohs 2018). Auch die Fortbildungsangebote in diesem Bereich sind noch immer sehr stark ausbaufähig (Ackeren et al. 2019). Die aktuellen bildungspolitischen Forderungen und Massnahmen zielen unter anderem auf die verpflichtende Verankerung medienpädagogischer Anteile im Lehramtsstudium (KMK 2016), obwohl die Frage nach Kompetenzen von Lehrpersonen zum Umgang mit (digitalen) Medien und nach dem lernförderlichen Einsatz ebendieser im Unterricht in Deutschland schon seit den 1990er Jahren diskutiert und mit bildungspolitischen Forderungen (u.a. KMK 1998) untermauert wird. Medienbildung ist zudem seit 2004 als ein Schwerpunkt der Vereinbarung über Standards in der Lehrerbildung ausgewiesen (KMK 2014). Eine flächendeckende Lehrerbildung in diesem Kontext ist jedoch noch nicht realisiert. Vor diesem Hintergrund beschäftigt sich der vorliegende Beitrag mit theoretischen Ansätzen zur systematischen Beschreibung der Kompetenzen von Lehrpersonen zum Einsatz digitaler Medien im Unterricht. Dazu werden drei Ansätze zur Beschreibung professioneller Handlungskompetenzen von Lehrpersonen in vergleichender Weise betrachtet und vor der Frage möglicher Implikationen für die Lehrerbildung diskutiert. Ergänzend werden im Rahmen der durch die Deutsche Forschungsgemeinschaft (DFG) geförderten Studie «Entwicklung der medienpädagogischen Kompetenz 
von Lehramtsstudierenden im Kontext der Digitalisierung in der Schule» (EmpädKLA) Einschätzungen von Lehramtsstudierenden hinsichtlich der Potenziale der Modelle ergänzt.

Theoretische Grundlagen und Überblick über zentrale empirische Befunde im Bereich professioneller Handlungskompetenzen von Lehrpersonen im Kontext der Digitalisierung

Hinsichtlich des Wissens, das Lehrpersonen benötigen, um im Kontext der Digitalisierung in der Schule professionell handeln und unterrichtliche Lehrprozesse gestalten sowie Lernprozesse anleiten zu können, liegen verschiedene theoretische Modelle und Ansätze vor. Diese sind in nationalen Kontexten entstanden und lassen sich wiederum auf unterschiedliche Traditionen und Verständnisse des professionellen Handelns von Lehrpersonen bzw. des Wissens und Handelns im Medienzusammenhang zurückführen. So hebt der auf Søby (2003) zurückgehende holistische Ansatz von «digital Bildung» insbesondere die reflexive Auseinandersetzung mit digitalen Medien für die persönliche Entwicklung hervor. Daran lehnt sich auch beispielsweise das österreichische Modell digi.KompP (Brandhofer et al. 2016) an, das den Kompetenzerwerb von Pädagoginnen und Pädagogen in acht Kategorien beschreibt, die sich über eine voruniversitäre Phase, das Studium und die ersten fünf Praxisjahre erstrecken. Die Kategorien umfassen allgemeine digitale Kompetenzen, fachspezifische sowie Schulverwaltungskompetenzen.

Im Folgenden werden mit dem Ansatz der Medienpädagogischen Kompetenz (Blömeke 2000; Herzig 2004), dem Modell des Technological Pedagogical And Content Knowledge (TPACK; Koehler und Mishra 2009; Mishra und Koehler 2006), sowie dem Europäischen Rahmen für die Digitale Kompetenz Lehrender (DigCompEdu; Redecker 2017) drei ausgewählte Ansätze näher beschrieben, die in Deutschland sowie im internationalen Diskurs massgebend sind. Diese theoretischen Konstrukte medienbezogener Wissens- und Kompetenzfacetten, die Lehrpersonen benötigen, um digitale Medien lernförderlich im Unterricht einsetzen zu können, sind auch Bestandteil empirischer Forschungsarbeiten, wobei die Intensität der empirischen Auseinandersetzung mit den Modellen stark variiert.

\section{Medienpädagogische Kompetenz}

Mit der Medienpädagogischen Kompetenz (Blömeke 2000; Herzig 2004) liegt ein in Deutschland auf Basis kommunikationstheoretischer Ansätze entwickeltes Modell vor, das zusätzlich zum kompetenten Umgang der Lehrpersonen mit (digitalen) Medien und deren lernförderlichen Verwendung im Unterricht weitere Kompetenzfacetten umfasst, die verstärkt auch schulische sowie gesellschaftliche Entwicklungsprozesse 
in den Blick nehmen. Insgesamt wird das Kompetenzgefüge, das Lehrpersonen für das Unterrichten mit und über (digitale) Medien benötigen, in fünf Kompetenzfacetten untergliedert (Blömeke 2000; s. Abbildung 1):

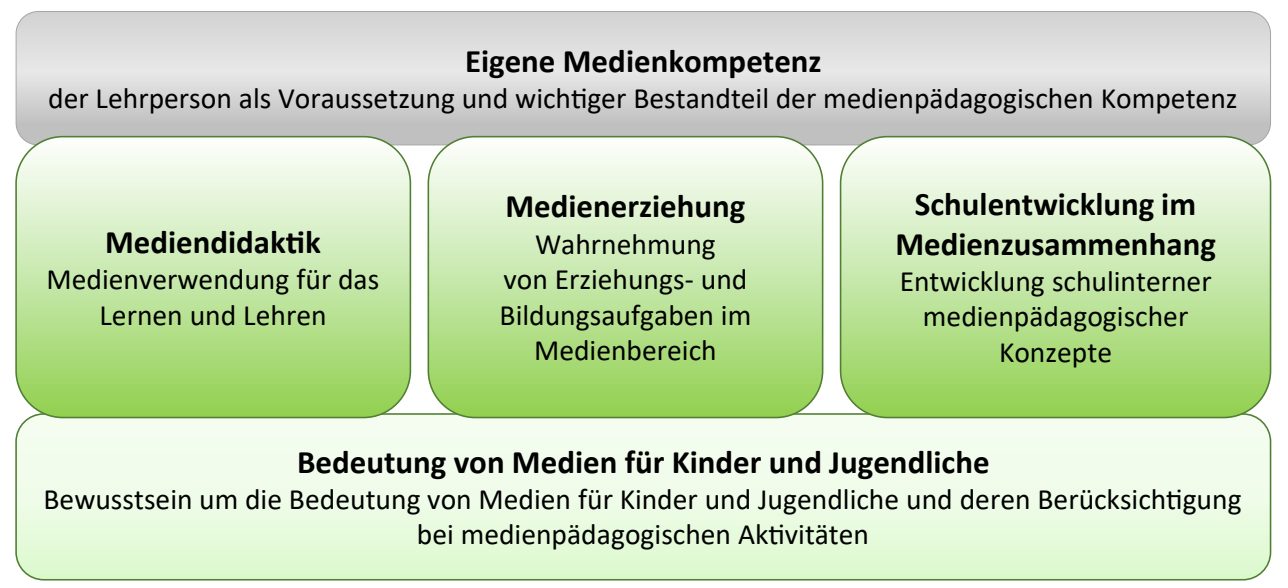

Abb. 1.: Medienpädagogische Kompetenzbereiche (eigene Darstellung nach Blömeke 2000).

Unterschieden werden (1) die eigene Medienkompetenz, als «Fähigkeit zu sachgerechtem, selbstbestimmtem, kreativem und sozial-verantwortlichem Handeln im Zusammenhang mit Medien und Informationstechnologien» (ebd., 172), (2) die mediendidaktische Kompetenz als «Fähigkeit zur reflektierten Verwendung von Medien und Informationstechnologien in geeigneten Lehr- und Lernformen und deren Weiterentwicklung» (ebd., 157), (3) die medienerzieherische Kompetenz als «Fähigkeit, Medienthemen im Sinne pädagogischer Leitideen im Unterricht behandeln zu können» (ebd., 159), (4) die sozialisationsbezogene Kompetenz im Medienzusammenhang als «Fähigkeit zur konstruktiven Berücksichtigung der Lernvoraussetzungen beim medienpädagogischen Handeln» (ebd., 162) und schliesslich (5) die schulentwicklungsbezogene Kompetenz im Medienzusammenhang als «Fähigkeit zur innovativen Gestaltung der Rahmenbedingungen medienpädagogischen Handelns» (ebd., 165). Die Darstellung orientiert sich dabei an der jüngeren thematisch-einschlägigen Literatur, in der die eigene Medienkompetenz von Lehrpersonen als Voraussetzung für den Medieneinsatz betrachtet und die sozialisationsbezogene Kompetenz als querliegend zu den weiteren Kompetenzbereichen verstanden wird (u.a. Tulodziecki 2012; Herzig und Martin 2018).

Empirische Evidenzen für die Reliabilität und Validität der angenommen fünf Kompetenzfacetten sind kaum vorhanden. Blömeke (2000) konnte im Rahmen einer exemplarisch an einer Universität durchgeführten Untersuchung die zunächst theoretisch differenzierten Kompetenzbereiche auch empirisch abbilden; eine erneute empirische Überprüfung des gesamten Modells ist bisher jedoch nicht erfolgt. Mit dem Projekt « $\mathrm{M}^{3} \mathrm{~K}$ - Modellierung und Messung der medienpädagogischen Kompetenz» 
wurde 2012 ein Versuch der theoretischen und empirischen Validierung eines Kompetenzstrukturmodells der Medienpädagogischen Kompetenz unternommen, in dem allerdings nur drei der fünf Kompetenzfacetten berücksichtigt wurden (Herzig et al. 2015). Die Ergebnisse einer ersten Pilotstudie mit 434 Lehramtsstudierenden konnten die zuvor durch Experten bescheinigte inhaltliche Validität der berücksichtigten Kompetenzaspekte empirisch jedoch nicht bestätigen. Vor dem Hintergrund des langen Bestehens des Ansatzes sowie der in der Medienpädagogik «seit mehreren Jahren mal mehr, mal weniger intensive[n] Diskussionen» (Schiefner-Rohs 2018, 56) um das Thema der Verankerung digitaler Medien in der Lehrerbildung, ist die fehlende empirische Absicherung als deutlicher Mangel des Konzepts aufzuzeigen.

\section{TPACK}

Ein international etablierter und im Gegensatz zum zuvor dargestellten Modell vielfach empirisch erforschter Ansatz zur Beschreibung des professionellen Wissens von Lehrpersonen zur Nutzung digitaler Medien im Unterricht besteht mit dem Ansatz TPACK (s. Abbildung 2; Hering et al. 2016; Koehler und Mishra, 2009; Voogt et al. 2013). TPACK fokussiert die Frage, wie Technologien und (digitale) Medien so in Lehr- und Lernprozesse integriert werden können, dass Fachinhalte entsprechend den gegebenen pädagogischen Anforderungen sowie in Anbetracht der kontextuellen Bedingungen möglichst einfach und verständlich vermittelt werden können. Dabei basiert TPACK auf den essentiellen Professionswissensfacetten «Fachwissen», «pädagogisches Wissen» und «fachdidaktisches Wissen» (Baumert und Kunter 2006), wobei letzteres von Shulman $(1986,1987)$ als Pedagogical Content Knowledge (PCK) den Kern des Professionswissens von Lehrpersonen darstellt, und erweitert diese um die Komponente des technischen Wissens (TK). 


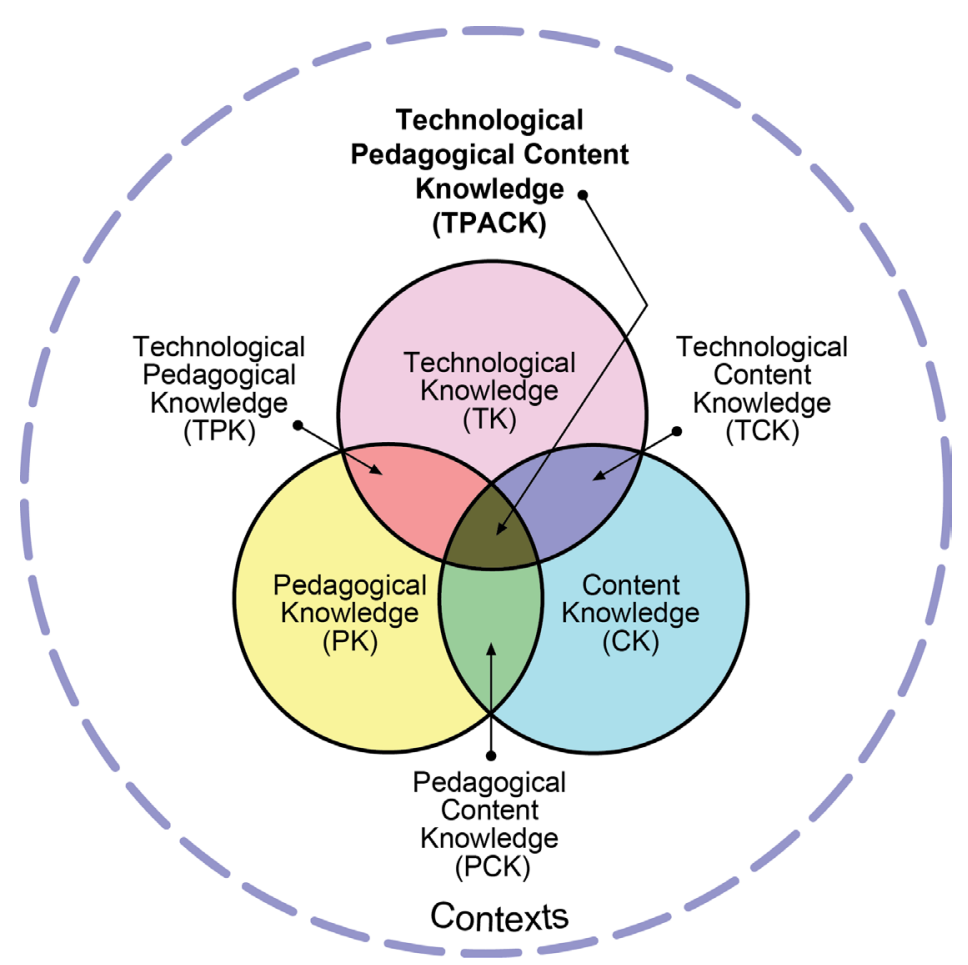

Abb. 2.: Das TPACK Modell (reproduced by permission of the publisher, (c) 2012 by tpack.org).

Das Wissen, das mit TPACK beschrieben wird, kann somit als essentieller Bestandteil der professionellen Handlungskompetenz von Lehrpersonen angesehen werden und sollte bereits im Rahmen der Lehrerausbildung erworben sowie über die Wahrnehmung professioneller Lerngelegenheiten in Form von Weiterbildungen über den gesamten Zeitraum der Berufsausübung vertieft und erweitert werden (u.a. Koehler et al. 2014; Niess 2013).

Einen ersten Zugang zu dem sehr breiten internationalen Forschungsfeld rund um TPACK bieten Forschungsüberblicke, in denen insbesondere die unterschiedlichen theoretischen und empirisch-methodischen Fragen rund um TPACK in den Blick genommen werden (u.a. Voogt et al. 2013; Willermark 2017). Ein Schwerpunkt der TPACK-Forschung besteht in der Frage nach dem Erwerb und Ausbau des entsprechenden Wissens im Rahmen der Lehreraus- und -fortbildung. Theoretische Arbeiten beschäftigen sich sowohl mit dem TPACK-Ansatz an sich (u.a. Graham 2011; Kimmons 2015) und setzen diesen auch in Verhältnis zu weiteren Modellen und theoretischen Zugängen (u.a. Niess und Gillow-Wiles 2017; Valtonen et al. 2015), sodass die wissenschaftliche Auseinandersetzung mit TPACK als sehr offen und selbstkritisch bezeichnet werden kann.

In Deutschland ist der Diskurs um TPACK erst vor wenigen Jahren angestossen worden und ist - nicht zuletzt durch die Berücksichtigung des Kernbereichs des TPACK-Modells für die Selbsteinschätzung medienbezogener Kompetenzen von 
Lehrpersonen im Rahmen der Untersuchung «Schule digital - der Länderindikator» (Endberg 2019; Endberg und Lorenz 2016; 2017) - mittlerweile grösstenteils in der nationalen wissenschaftlichen Community als etabliert einzustufen (Aktionsrat Bildung 2018). Dennoch liegen in Deutschland trotz der internationalen Verbreitung des TPACK-Modells und der breiten empirischen Grundlage bisher kaum Ansätze vor, TPACK systematisch in die Lehrerausbildung zu implementieren und die professionellen Kompetenzen von Lehrpersonen auch an der Schnittstelle zwischen Fachinhalten, Lehrmethoden und Technologien gezielt zu fördern. Als inhaltliches Manko des TPACK Ansatzes lässt sich festhalten, dass die Zieldimension des Outputs in Form der Medienkompetenz der Schülerinnen und Schüler nicht explizit im Modell benannt ist und im bisherigen Forschungsdiskurs zumeist ausser Acht gelassen wird. Der TPACK-Ansatz konzentriert sich hauptsächlich auf die schulische Prozessebene. Die (scheinbare) Einfachheit des Modells ermöglicht die niedrigschwellige Anwendung, kaschiert aber auch die komplexen Möglichkeiten, die TPACK für die Unterrichtsgestaltung eröffnet. Schliesslich liegt mit TPACK ein Ansatz vor, der in direkter Anbindung an die Konzeptionen zur Bestimmung des Lehrerprofessionswissens entwickelt wurde und damit das Kerngeschäft der Lehrpersonen - das Unterrichten - klar in den Vordergrund rückt.

\section{DigCompEdu}

Von der Europäischen Kommission wurde 2017 der European Framework for the Digital Competence of Educators (kurz: DigCompEdu) entwickelt, der die spezifischen digitalen Kompetenzen, die Lehrende aller Bildungsstufen im Rahmen ihrer Tätigkeit benötigen, komprimiert. Der Referenzrahmen kann und soll dazu genutzt werden, die Entwicklung von (nationalen) digitalen Kompetenzmodellen zu unterstützen.

Er wurde unter Zusammenarbeit von Lehrpersonen, Wissenschaftlerinnen und Wissenschaftlern sowie weiterer Expertinnen und Experten im Rahmen von Workshops und Online-Konsultationen entwickelt und umfasst sechs Bereiche. Diese differenzieren die professionsbezogenen und pädagogischen Kompetenzen der Lehrenden und legen zudem besonderes Augenmerk auf die Förderung der Kompetenzen Lernender (Redecker 2017; s. Abbildung 3). 


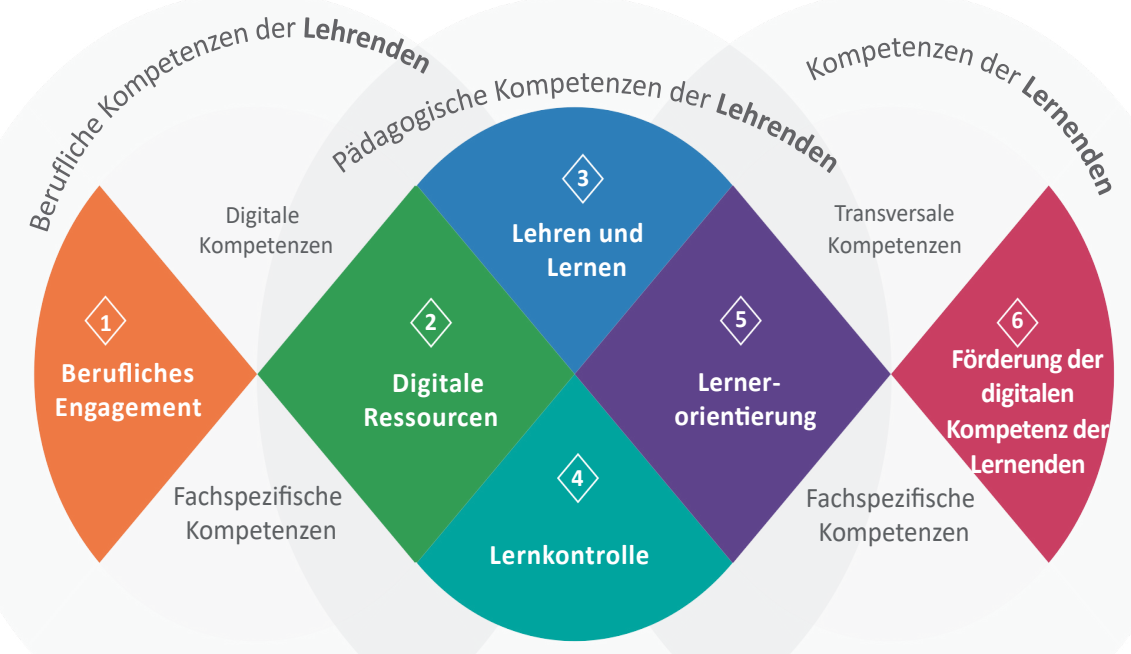

Abb. 3.: Das DigCompEdu-Modell (nach Redecker 2017).

Das (1) Berufliche Engagement umfasst die Nutzung digitaler Medien für die berufsbezogene Kommunikation, Kooperation und professionelle Weiterbildung, der Bereich (2) Digitale Ressourcen bezieht sich auf die Auswahl, Erstellung und Verbreitung digitaler Ressourcen, (3) Lehren und Lernen umfasst die Organisation und Inszenierung der Nutzung digitaler Medien und Ressourcen in Lehr- und Lernkontexten, die (4) Lernkontrolle bezieht sich auf die Verwendung digitaler Medien und Strategien für die Planung, Rückmeldung und Kontrolle von Lernprozessen und Lernergebnissen, die (5) Lernerorientierung beschreibt die Verwendung digitaler Medien für die Organisation eines inklusiven, personalisierten und aktiv involvierenden Unterrichts und (6) die Förderung der digitalen Kompetenzen der Lernenden versteht sich als Anleitung der Schülerinnen und Schüler zu einer kreativen und verantwortlichen Nutzung digitaler Medien, z.B. für die Informationsrecherche, Kommunikation, Erstellung eigener Inhalte und das gezielte Problemlösen. Die sechs Kompetenzbereiche sind in insgesamt 22 Kompetenzen unterteilt. Der Referenzrahmen ermöglicht es zudem durch festgelegte Standards pro Kompetenzbereich eine Einschätzung der vorhandenen Kompetenzen von Lehrenden vorzunehmen und gemäss eines Kategoriensystems einzuordnen. Dieses Kategoriensystem ist in Anlehnung an den Gemeinsamen Europäischen Referenzrahmen für Sprachen (GER) entwickelt worden und bietet somit die Möglichkeit, den Kompetenzstand entsprechend der bewährten Einordnung von A1 zu C2 festzulegen (Redecker 2017). Langfristig ist geplant, dass Lehrende aller Bildungsstufen in Europa ihren Kompetenzstand mittels eines Online-Selbsteinschätzungsinstruments entlang der beschriebenen Niveaustufen ermitteln können. Eine erste Pilotierung des Selbsteinschätzungsinstruments mit 335 Lehrpersonen in 
Deutschland deutet auf eine akzeptable Reliabilität und Validität des Instruments insgesamt sowie seiner sechs Teilkonstrukte hin (Ghomi und Redecker 2019).

Die inhaltliche Breite des DigCompEdu-Modells wird durch die strukturelle Einteilung in die drei Bereiche «berufliche Kompetenzen der Lehrenden», "Pädagogische Kompetenzen der Lehrenden» und «Kompetenzen der Lernenden» verdeutlicht. Zu betonen ist, dass die Förderung der digitalen Kompetenzen der Lernenden explizit als Kompetenzbereich ausgewiesen ist. Zudem ist die Kompetenz, mithilfe digitaler Medien den Lernstand der Lernenden zu erheben, Lernverhalten zu analysieren und Feedback zu geben, als eigenständiger Bereich hinterlegt. Mit der gezielten Ausgestaltung des Modells als Selbstdiagnoseinstrument ist zudem eine praktische Anwendbarkeit zu verzeichnen, die sich in veränderten professionellen Handlungen der Lehrpersonen widerspiegeln kann.

\section{Vergleich der Ansätze}

Ein Vergleich der genannten Ansätze kann in unterschiedlicher Form vorgenommen werden. Es könnten beispielsweise die Vergleichsebenen der Entstehungszusammenhänge, der wissenschaftlichen Bezugsdisziplinen, der intendierten Zielgruppe(n) sowie der Zielsetzungen herangezogen werden. Letztere stellt den Ausgangspunkt für den Modellvergleich im vorliegenden Beitrag dar. Dafür soll insbesondere die Frage im Vordergrund stehen, welche Potenziale und Implikationen aus den Modellen für die Lehrerbildung abgeleitet werden können. Hervorzuheben ist, dass der Beitrag nicht den Anspruch erhebt, die im Titel formulierten «Professionellen Handlungskompetenzen von Lehrpersonen im Kontext der Digitalisierung in der Schule» durch die drei hier vorgestellten Modelle in Gänze zu beschreiben. Dennoch weisen der Ansatz der Medienpädagogischen Kompetenz, TPACK sowie der Kompetenzrahmen DigCompEdu im Einzelnen sowie gemeinsam betrachtet viele ebendieser Handlungskompetenzen aus. Dabei ist es wichtig im Blick zu behalten, dass mit «professionellen» Kompetenzen ein direkter Bezug zum Diskurs um das Professionswissen von Lehrpersonen (u.a. Baumert und Kunter 2006) angestrebt wird und die «Digitalisierung» als andauernder gesamtgesellschaftlicher Veränderungsprozess sowie als notwendiger Anlass für Schulentwicklungsprozesse verstanden wird (Cress et al. 2018).

Gemeinsam ist allen drei Modellen, dass Wissensbereiche bzw. Kompetenzen definiert werden, die sich auf folgende Bereiche beziehen:

- das Wissen und die Erfahrung von Lehrpersonen im Umgang mit Technologien und digitalen Ressourcen,

- die pädagogisch-didaktischen Kompetenzen der Lehrpersonen zur Gestaltung von mediengestütztem Unterricht sowie

- das Wissen um die Lernvoraussetzungen der Schülerinnen und Schüler. 
Dabei ist bezüglich des Wissens und der Erfahrung von Lehrpersonen im Umgang mit Technologien und digitalen Ressourcen festzustellen, dass die eigene Medienkompetenz im Ansatz der Medienpädagogischen Kompetenz als Voraussetzung für die Handlungsfähigkeiten der Lehrpersonen in den weiteren Kompetenzbereichen verstanden wird (Abbildung 1), während «technological knowledge» (TK) in TPACK und das Wissen um «Digitale Ressourcen» in DigCompEdu mit den pädagogisch-didaktischen Kompetenzen gleichberechtigt auf einer Ebene gesehen werden (Abbildung 2 und Abbildung 3). Aus der internationalen Forschung lassen sich Hinweise dazu ableiten, dass der separate Erwerb von medien-/technologiebezogenem Wissen im Rahmen des Lehramtsstudiums sowie im Kontext von Lehrerfortbildungen nicht zielführend ist (u.a. Kushner-Benson et al. 2015; Mouza 2016). Mit Blick auf die Lehrerbildung stellen die diesbezüglichen Modellannahmen in TPACK und DigCompEdu eher die Konzeption von Lerngelegenheiten in Aussicht, die auf einen pädagogischdidaktisch abgestimmten Medieneinsatz vorbereiten. Für TPACK liegen dazu bereits bestätigende Forschungsbefunde, z.T. mit längsschnittlich erhobenen Daten, im Rahmen der Lehrerausbildung vor.

Im Hinblick auf die durch alle Modelle in den Blick genommenen pädagogischdidaktischen Kompetenzen der Lehrpersonen zur Gestaltung von mediengestütztem Unterricht ist festzustellen, dass diese im Rahmen von DigCompEdu am differenziertesten betrachtet werden. Hierunter können sowohl der Kompetenzbereich «Lehren und Lernen» als auch die schülerzentrierten Kompetenzbereiche «Lernkontrolle», «Lernerorientierung» gezählt werden. Der Bereich des Lehrens und Lernens mit digitalen Medien ist unter anderen Bezeichnungen auch in den beiden weiteren Modellen berücksichtigt (Mediendidaktische Kompetenz bzw. TCK, TPK, TPACK). In DigCompEdu werden dabei allerdings zusätzlich auch die Erhebung und Analyse lernrelevanter Daten sowie die Bereitstellung von Feedback und die Möglichkeiten des Einsatzes digitaler Medien zur Differenzierung und Individualisierung sowie aktiven Einbindung der Lernenden angesprochen. Für die Lehrerbildung ergeben sich daraus vielzählige Potenziale der Gestaltung von Lerngelegenheiten, zum einen in Form von einzelnen Lehrveranstaltungen zu den einzelnen Schwerpunkten, zum anderen aber auch in Form von Studienmodulen oder Fortbildungsreihen, die aufeinander aufbauend und miteinander verknüpfend den Erwerb und Ausbau der pädagogisch-didaktischen Kompetenzen von Lehrpersonen im Kontext der Digitalisierung fördern. Mit Blick auf die Ansätze der Medienpädagogischen Kompetenz und TPACK scheint dieses Potenzial sehr viel eingeschränkter gegeben. Da der Bereich der Mediendidaktischen Kompetenz nicht weiter ausdifferenziert wird, scheint das Risiko gross, dass Lehrveranstaltungen und Fortbildungen unter diesem Label zu oberflächlich konzipiert werden und beispielsweise nur Fragen der Auswahl von Medienangeboten für den Unterricht nach lernrelevanten Kriterien im Vordergrund stehen und Fragen der Anpassung und Erweiterung der Unterrichtsmethoden aussenvorgelassen werden. Im 
Fall von TPACK ist eine Differenzierung in verschiedene Wissensbereiche, die Fragen rund um das Lehren und Lernen mit digitalen Medien fokussieren, gegeben. Allerdings offenbart die umfangreiche TPACK-Forschung die fehlende empirische Trennschärfe zwischen den einzelnen Wissensbereichen, sodass hier ähnlich wie für die Medienpädagogische Kompetenz die gezielte Aufbereitung von Lerngelegenheiten im Rahmen der Lehrerbildung schwierig erscheint. Zudem herrscht bisher keine Einigkeit darüber, wie der Erwerb der Wissensbestände, die in TPACK vereint sind, am besten erfolgen kann (u.a. Koehler et al. 2014).

Die dritte allen Modellen gemeinsame Übereinstimmung besteht in der Berücksichtigung des Wissens der Lehrpersonen über die Lernvoraussetzungen der Schülerinnen und Schüler. Im Ansatz des DigCompEdu erfolgt dies über den bereits angesprochenen Wissensbereich der Lernerorientierung, der u.a. Fragen des Lernzugangs und der Inklusion, der Differenzierung und Personalisierung sowie der aktiven Beteiligung umfasst. In TPACK sind es insbesondere die Wissensbereiche PK und PCK, in denen die professionswissenstheoretisch hervorgehobene Kenntnis und Anwendung von Sozialisations- und Lerntheorien (PK) sowie fachdidaktisch relevante Aspekte, wie die Berücksichtigung des fachbezogenen Vorwissens, typischer Fehler von Schülerinnen und Schülern unterschiedlichen Lernstandes sowie Lernschwierigkeiten (PCK), betont werden. Damit zeigen sich DigCompEdu und TPACK weitestgehend anschlussfähig an die in den Standards für die Lehrerbildung ausgewiesenen Kompetenzbereiche «Unterricht» und «Erziehung» (KMK 2014). Im Fall der Medienpädagogischen Kompetenz bezieht der Bereich der sozialisationsbezogenen Kompetenz im Medienzusammenhang die Lernvoraussetzungen der Schülerinnen und Schüler im Medienkontext mit ein, jedoch nicht aus lehr- und lerntheoretischer Perspektive. Führt man sich die kommunikationstheoretischen Grundlagen und die Entwicklung des Ansatzes aus dem Medienkompetenzdiskurs vor Augen, ist dies nicht verwunderlich und erklärt, warum der Ansatz der Medienpädagogischen Kompetenz als einziges der drei Modelle den expliziten Medienbezug in allen Kompetenzfacetten ausweist. Gerade vor der Betrachtung der Digitalisierung als gesamtgesellschaftlichem Veränderungsprozess und der im Ansatz ausgewiesenen Perspektive der Förderung der Medienkompetenz der Schülerinnen und Schüler als Ziel des medienpädagogischen Handelns der Lehrpersonen (Blömeke 2000), ist die Relevanz dieses Kompetenzbereichs jedoch nicht zu verachten und sollte im Rahmen der Lehrerbildung zumindest implizit Berücksichtigung finden.

\section{Die Perspektive der Lehramtsstudierenden}

Der Vergleich ausgewählter Modelle zur Bestimmung der professionellen Handlungskompetenzen von Lehrpersonen im Kontext der Digitalisierung in der Schule und die Frage nach möglichen Implikationen für die Lehrerbildung wird an dieser Stelle um 
die Perspektive von Lehramtsstudierenden, die sich im Rahmen einer Lehrveranstaltung mit den Modellen auseinandergesetzt haben und diese für die Konzeption und Erprobung exemplarischer Unterrichtskonzepte herangezogen haben, ergänzt. Mit der durch die Deutsche Forschungsgemeinschaft (DFG) geförderten Studie «Entwicklung der Medienpädagogischen Kompetenz von Lehramtsstudierenden im Kontext der Digitalisierung in der Schule» (EmpädK-LA) wird das Ziel verfolgt, das Professionswissen, die professionsbezogenen Wissenseinschätzungen sowie die Kompetenzen zur Planung und beispielhaften Durchführung von Unterrichtseinheiten von Lehramtsstudierenden jeweils unter spezifischer Berücksichtigung des Kontexts der Digitalisierung in der Schule gezielt zu fördern und empirisch auszuwerten. Im Rahmen der Lehrveranstaltung wurden den Studierenden die Ansätze der Medienpädagogischen Kompetenz, DigCompEdu sowie TPACK und das sogenannte «TPACKGame» (Endberg et al. 2018, 26) vorgestellt und diskutiert. Im Anschluss an die Seminarsitzungen bekamen die Studierenden Reflexionsaufgaben, die eine vertiefte, textgestützte Auseinandersetzung mit den Modellen ermöglichen sollte. Anhand der schriftlich durch die Studierenden ausgearbeiteten Reflexionsfragen war es möglich, die von den Studierenden identifizierten Potenziale der drei Modelle inhaltsanalytisch auszuwerten. Dazu lagen die schriftlichen Antworten von 32 Studierenden vor. Die Lehrveranstaltung war im verpflichtenden Studienanteil der Bildungswissenschaften im Bachelor des Lehramts der TU Dortmund angelegt. An dem Seminar nahmen Studierende unterschiedlicher Lehramtsstudiengänge teil. Der Grossteil der Studierenden befand sich zu Beginn des Wintersemesters 2018/2019 im zweiten Bachelorfachsemester. Den Studierenden war vor Beginn der Lehrveranstaltungen keines der Modelle bekannt.

Die schriftlichen Ausarbeitungen wurden hinsichtlich der Frage ausgewertet, welche Potenziale die Studierenden den Modellen zuschreiben. Dabei wurde in Form einer quantitativen Auswertung gezählt, wie oft welches Potenzial für welches Modell benannt wurde. Die einzelnen Kategorien der Potenziale wurden dabei rein induktiv aus den schriftlichen Ausarbeitungen gebildet.

Es zeigt sich, dass die Studierenden in den Modellen insgesamt insbesondere Potenziale für die Gestaltung und Weiterentwicklung von Unterricht benennen. Besonders sticht hier das TPACK-Modell hervor, dem die Mehrheit der Studierenden besonderen Nutzen aus pädagogisch-didaktischer Sicht zuspricht. Vor allem wird die Fokussierung auf die etablierten Kernkomponenten des Professionswissens von Lehrpersonen als besonders positiv hervorgehoben. Dies steht auch in engem Zusammenhang mit der allein im TPACK-Modell explizit berücksichtigten Kombination der Wissensbereiche, die von den Studierenden ebenfalls als äusserst nützlich angesehen wird. Im Einzelnen verweisen die Studierenden darauf, dass durch die notwendige Abstimmung von Fachinhalten, pädagogischen Lehr- und Lernmethoden und dem technischen Wissen im Kernbereich TPACK einer zu starken Technikzentrierung 
bzw. einem pädagogisch und fachlich nicht sinnvollem Einsatz digitaler Medien vorgebeugt werden kann. Doch auch den beiden anderen Modellen sprechen die Studierenden deutliche Potenziale für die Gestaltung und Weiterentwicklung von Unterricht zu. In diesem Sinne erkennen sie ebenfalls die zuvor im Modellvergleich ausgeführte Gemeinsamkeit der Modelle in der Betonung der pädagogisch-didaktischen Kompetenzen von Lehrpersonen. Interessant ist an dieser Stelle, dass die Studierenden die in den Modellen ausgewiesenen Kompetenzen in ihren Ausarbeitungen praxisorientiert reflektieren und direkte Bezugspunkte zu ihrem zukünftigen Berufsalltag aufzeigen. Dies unterstreicht die Relevanz und Anwendbarkeit der Modelle im Kontext der Lehrerausbildung.

Ein weiteres oft genanntes Potenzial ist die Kompetenzerweiterung, welche die Studierenden ebenfalls in allen drei Modellen erkennen - in erster Linie jedoch im Ansatz des DigCompEdu. Durch die Konzeption als Referenzrahmen mit fest definierten Kompetenzbereichen und den Bemühungen um die Etablierung der Niveaustufen ist die häufige Zuschreibung dieses Potenzials zu DigCompEdu nicht überraschend. Die Studierenden beschreiben insbesondere die Gelegenheit, sich selbst auf einer der vorgegebenen Kompetenzstufen verorten zu können, als hilfreich für ihre zukünftige berufliche Praxis. Einige sehen zudem den Vorteil, dass Lehrpersonen auf höheren Kompetenzstufen, andere Lehrpersonen anleiten können; sie zeigen also Möglichkeiten des Aufbaus von Kooperationen und professionellen Lerngemeinschaften auf. Der von den Studierenden benannte Vorteil der Kompetenzerweiterung deckt sich mit der im Modellvergleich herausgearbeiteten Betonung des Wissens und der Erfahrung von Lehrpersonen mit digitalen Medien, geht aber über die in den Modellen explizit genannten entsprechenden Kompetenzen hinaus. Auch hier ist der starke Praxisbezug, der die Studierendenperspektive prägt, zu erkennen. Einer separaten Betrachtung von Medienkompetenz, wie sie in den Modellen verortet ist, wird kaum Bedeutung beigemessen.

Auf die dritte im Modellvergleich beschriebene Gemeinsamkeit - der Betonung des Wissens um die Lernvoraussetzungen der Schülerinnen und Schüler - wird seitens der Studierenden kaum eingegangen. Es finden sich einige Aussagen der Studierenden, die die Schülerorientierung der Modelle positiv hervorheben, es wird jedoch an keiner Stelle gezielt auf die Berücksichtigung des Vorwissens verwiesen. Denkbar ist aber, dass die Studierenden dies implizit als relevanten Wissens- und Kompetenzbereich verstehen, der für die Gestaltung und Weiterentwicklung von Unterricht als elementar angesehen wird.

Die Betrachtung der Potenziale, die den einzelnen Modellen durch die Studierenden zugeschrieben werden, zeigt folgendes Bild: In Bezug auf DigCompEdu weisen die Studierenden insbesondere auf die inhaltliche Breite der Kompetenzbereiche hin, die aus ihrer Sicht von allen Modellen am ehesten eine Bildung in der digitalen Welt repräsentieren. Im Gegensatz dazu wird dem TPACK-Modell seine Einfachheit 
als Vorteil zugesprochen: es eignet sich den Studierenden zufolge von den drei Modellen am besten für einen niedrigschwelligen Zugang zu der Frage, wie digitale Medien lernförderlich im Unterricht eingesetzt werden können, da das fachdidaktische Wissen der Lehrpersonen in den Mittelpunkt gestellt wird.

Dem Modell der Medienpädagogischen Kompetenz, das von den wenigsten Studierenden als präferiertes Modell ausgewählt wurde, wird zumindest von einer kleinen Anzahl Studierender die explizite Berücksichtigung der Schulebene als besonderes Potenzial zugesprochen.

Insgesamt zeigen sich die Studierenden allen drei Modellen gegenüber aufgeschlossen und sprechen darüber hinaus allen Ansätzen eine hohe Praxistauglichkeit zu. Ausserdem sehen sie in allen Modellen das bildungspolitisch geforderte «Primat des Pädagogischen» (KMK 2016, 9) als gegeben.

\section{Diskussion}

Einig ist man sich seitens der Bildungspolitik und der Bildungsforschung darin, dass die Digitalisierung neue Anforderungen für Schulen und insbesondere für Lehrpersonen mit sich bringt. Worin allerdings noch kein Konsens gefunden wurde, ist ein theoretischer Rahmen, der die professionellen Handlungskompetenzen von Lehrpersonen im Kontext der Digitalisierung umfassend beschreibt und empirisch abgesichert ist. Entsprechend fehlen Orientierungen für die Lehrerbildung. Die aus wissenschaftlicher Perspektive geführte Diskussion der Ansätze wird um die Sichtweise der Lehramtsstudierenden erweitert, die insbesondere die Praxistauglichkeit der Modelle kritisch in den Blick nehmen. Dabei kann dieser Beitrag lediglich einen ersten Zugang zur Schliessung der aufgezeigten Forschungslücke darstellen, der im Rahmen der Untersuchung «EmpädK-LA» in qualitativ-exploratorischer Herangehensweise erstmalig die drei Modelle Medienpädagogische Kompetenz, TPACK und DigCompEdu theoretisch zusammenführt sowie exemplarisch in die Lehramtsausbildung integriert.

Mit dem Vergleich ausgewählter etablierter und für die internationale Anschlussfähigkeit relevanter Ansätze lässt sich zusammenfassend festhalten, dass jedes der drei Modelle seine individuellen Vorzüge aufweist und sich somit legitimiert, im Kontext der Lehrerbildung berücksichtigt zu werden. Der Ansatz der Medienpädagogischen Kompetenz zeichnet sich insbesondere durch die inhaltliche Breite sowie die explizite Berücksichtigung der Schulentwicklungskompetenz aus, die im Übrigen auch durch die Studierenden, die diesem Ansatz ansonsten eher skeptisch gegenüber eingestellt waren, als positives Merkmal hervorgehoben wird.

Das besondere Kennzeichen von TPACK besteht in der zusammenführenden Betrachtung von fachlichem, pädagogischem und technischem Wissen. Insbesondere in dieser Kombination der Wissensbereiche erkennen die Studierenden einen klaren 
Vorteil des TPACK-Modells gegenüber den beiden weiteren Ansätzen. Vergleicht man TPACK mit dem in Deutschland verbreiteten Ansatz der Medienpädagogischen Kompetenz von Lehrkräften, zeigen sich Überschneidungen der beiden Ansätze in den Bereichen der Medienkompetenz der Lehrpersonen (im Sinne der sicheren und reflektierten Handhabung digitaler Medien) und der didaktischen Kompetenzfacette.

Im Rahmen des DigCompEdu wird die Betonung auf die pädagogisch-didaktischen Kernkompetenzen sowie die eigene Medienkompetenz von Lehrpersonen, wie sie auch in beiden zuvor herangezogenen Modellen erfolgt, um die beruflichen Kompetenzen ergänzt. Zudem wird die Förderung der digitalen Kompetenzen der Lernenden einzig in DigCompEdu explizit als Kompetenzbereich ausgewiesen. Auffällig ist hier, dass die Studierenden die pädagogisch-didaktischen Kernkompetenzen des Modells positiv hervorheben, jedoch nur vereinzelt auf die beruflichen Kompetenzen sowie die Förderung der digitalen Kompetenzen der Lernenden eingehen. Bei der genauen Betrachtung der 22 Kompetenzen, die den sechs Kompetenzbereichen des DigCompEdu-Modells zugeordnet werden, finden sich viele Überschneidungen zu den im Rahmen der Medienpädagogischen Kompetenz oder TPACK berücksichtigten Kompetenzen. Aufgrund der bisher fehlenden empirischen Überprüfung des Modells kann ein valider Vergleich der Zuordnung der Kompetenzfacetten über die drei Modelle jedoch noch nicht vorgenommen werden.

Der Blick in den Forschungsstand offenbart zudem, dass bisher keines der Modelle im deutschsprachigen Kontext der Lehrerbildung bzw. der Lehrerkompetenzforschung als intensiv erforscht erachtet werden kann. Mit der Berücksichtigung des Kernbereichs des TPACK-Modells im Rahmen der Untersuchungen der Länderindikatoren 2016 und 2017 (Endberg 2019; Endberg und Lorenz 2016; 2017), konnten fundierte Kenntnisse über die Selbsteinschätzungen von Lehrpersonen in Deutschland in diesem Bereich generiert werden. Für die Personengruppe der Lehramtsstudierenden liegen jedoch bisher keine einschlägigen TPACK-bezogenen Befunde in Deutschland vor. Mit der Berücksichtigung von TPACK im Rahmen von Untersuchungen zur Lehrerausbildung in Deutschland wird der Vergleich mit anderen Lehrerausbildungssystemen möglich und die internationale Anschlussfähigkeit in der Lehrerausbildung damit gesichert. Mit dem Kompetenzrahmen DigCompEdu liegt ein bisher unerforschter Ansatz vor, dessen Nutzen für die Bildungsforschung sowie die Bildungspraxis noch ungeklärt ist. Da dieser Bezugsrahmen auf europäischer Ebene jedoch zukunftsweisend für die Kompetenzen von Lehrenden sein soll und der allgemein zur Beschreibung notwendiger digitaler Kompetenzen ausgelegte Ansatz DigComp (Ferrari 2013) auch in die KMK-Strategie zur Beschreibung der an Schülerinnen und Schüler zu vermittelnden «Kompetenzen in der digitalen Welt» eingeflossen ist (KMK 2016), erscheint es unverzichtbar, DigCompEdu bei der Konzeption eines umfassenden medienpädagogischen Wissens von Lehrpersonen zu berücksichtigen und damit einen innovativen Ansatz zu verfolgen. 
Der in diesem Beitrag erstmalig angestellte Vergleich der Modelle liefert Hinweise darauf, welche Aspekte der betrachteten Modelle besondere Anknüpfungspunkte für die Lehrerbildung bieten. Hierbei ist es in erster Linie relevant, sich auf die grundlegenden Kompetenzen, die professionelles Handeln von Lehrpersonen auszeichnen, zurückzubesinnen und diese entsprechend den Anforderungen in der digitalen Welt um weitere Komponenten zu erweitern. Hier sind insbesondere die Perspektive der gemeinsamen Betrachtung von inhaltlichen, pädagogischen und technischen Wissensfacetten im TPACK-Modell sowie die im pädagogisch-didaktischen Kern des DigCompEdu ausgewiesenen Kompetenzbereiche hilfreich. Gleichzeitig sollten sich die Lehrpersonen im Sinne des Bildungs- und Erziehungsauftrags auch der Förderung der digitalen Kompetenzen der Lernenden widmen, um diese im Sinne des verantwortlichen, sicheren und reflektieren Umgangs mit digitalen Medien(angeboten) zu schulen und damit auf die aktive Teilhabe am beruflichen, gesellschaftlichen und privaten Leben vorzubereiten. Diese Zielperspektive, die Ausgangspunkt der Entwicklung des Ansatzes der Medienpädagogischen Kompetenz war, wird im Modell des DigCompEdu explizit aufgegriffen und als eigenständiges Kompetenzfeld definiert. Aus Sicht der aktiv im Schuldienst tätigen Lehrpersonen kommt der Förderung der digitalen Kompetenzen der Schülerinnen und Schüler im Laufe der ersten beiden Lehrerausbildungsphasen allerdings noch nicht genug Bedeutung zu (Eickelmann et al. 2016).

\section{Ausblick}

Mit den Ansätzen der Medienpädagogischen Kompetenz, DigCompEdu und TPACK liegen Ansätze vor, die darauf ausgerichtet sind, die Wissensbestände bzw. Kompetenzen zu beschreiben, die Lehrpersonen im Kontext der Digitalisierung in der Schule benötigen. Dass diese in sich nicht erschöpfend sind, konnte durch die erfolgte Gegenüberstellung aufgezeigt werden. Doch auch die gezielte Kombination der Modelle scheint nicht ausreichend zu sein, um alle Anforderungen, denen Lehrpersonen in der schnelllebigen und hochkomplexen digitalisierten Welt gegenüberstehen, beschreiben zu können. Dies zeigt ein Blick in die «Kompetenzen in der digitalen Welt», die durch den Beschluss der KMK (2016) definiert worden sind und alle Schülerinnen und Schüler verpflichtend im Laufe der Pflichtschulzeit erwerben sollen. Damit dies gelingt, ist es notwendig, dass Lehrpersonen ebenfalls über diese Kompetenzen verfügen und darüber hinaus ihre schulischen Bildungs- und Erziehungsaufgaben dahingehend ausrichten, die Schülerinnen und Schüler gezielt hinsichtlich dieser Kompetenzen zu fördern. Zudem erfassen die Modelle bisher nicht explizit die Förderung von überfachlichen Kompetenzen wie beispielsweise der Kooperation, Methodenkompetenz oder Problemlösestrategien. Aktuell sind zudem rechtliche Fragestellungen rund um Datenschutz und Urheberrechte für Lehrpersonen relevant, 
die ebenfalls unberücksichtigt bleiben. Nicht zuletzt sollte ein ganzheitlicher Ansatz im Sinne von «Bildung» (angelehnt an Søby 2003) die persönliche Weiterentwicklung betonen und schliesslich auch Fähigkeiten umfassen, die die Bewältigung von schulischen Herausforderungen - als Beispiel kann die Inklusion oder individuelle Förderung genannt werden - mithilfe digitaler Medien einschliesst. Derzeit werden die Kompetenzstandards für Lehrpersonen durch die KMK überarbeitet. Wünschenswert wäre dabei, dass die pädagogisch-didaktischen Kernkompetenzen der Lehrpersonen, wie sie in TPACK durch die Anbindung der professionswissenstheoretischen Grundlagen und auch im Modell des DigCompEdu im pädagogisch-didaktischen Kern berücksichtigt sind, nicht aus den Augen verloren werden, um guten Unterricht, der alle Schülerinnen und Schüler erreicht und den Anforderungen der digitalisierten Welt gerecht wird, jetzt und in Zukunft gestalten zu können.

Kurz- bis mittelfristig besteht also ein Desiderat im Entwurf von Konzepten an lehrerbildenden Hochschulen zur Entwicklung einer umfassenden professionellen Handlungskompetenz im Kontext der Digitalisierung von Lehramtsstudierenden in den Fächern, Fachdidaktiken und Bildungswissenschaften. Mit dieser Verzahnung kann zur systematischen und aufeinander aufbauenden Kompetenzentwicklung beigetragen werden. Langfristig kann dann der Übergang in den Vorbereitungsdienst in den Blick genommen werden, in dem an zuvor erworbene Kompetenzen angeknüpft wird und mit der verstärkten schulischen Praxis die Entfaltung der Kompetenzen intensiviert werden kann. Schliesslich gilt es auch in der Fortbildung professionelle Handlungskompetenzen im Kontext der Digitalisierung zu vermitteln, damit einerseits der sinnvolle und lernförderliche Umgang mit digitalen Medien im Unterricht von bereits im Schuldienst tätigen Lehrkräften (weiter)entwickelt wird, andererseits aber auch bei medienpädagogisch ausgebildeten Lehrpersonen die Kompetenzen in Tiefe und Breite weiter ausgebaut werden, damit Innovationen in der Schul- und Unterrichtsentwicklung Potenziale entfalten können. Nur mit umfassend ausgebildeten Lehrpersonen können Schülerinnen und Schüler auf die Anforderungen des digitalen Zeitalters angemessen vorbereitet werden, womit einer digitalen Spaltung entgegengewirkt und die Chancengleichheit erhöht wird. 


\section{Literatur}

Ackeren, Isabell van, Stefan Aufenanger, Birgit Eickelmann, Steffen Friedrich, Rudolf Kammerl, Julia Knopf, Kerstin Mayrberger, Heike Scheika, Katharina Scheiter, und Mandy SchiefnerRohs. 2019. «Digitalisierung in der Lehrerbildung. Herausforderungen, Entwicklungsfelder und Förderung von Gesamtkonzepten». Die Deutsche Schule, 111(1): 103-119.

Aktionsrat Bildung. 2018. Digitale Souveränität und Bildung. Gutachten. Münster: Waxmann.

Baumert, Jürgen, und Mareike Kunter. 2006. «Stichwort: Professionelle Kompetenz von Lehrkräften». Zeitschrift für Erziehungswissenschaft 9 (4): 469-520. https://doi.org/10.1007/ s11618-006-0165-2.

Blömeke, Sigrid. 2000. Medienpädagogische Kompetenz. Theoretische und empirische Fundierung eines zentralen Elements der Lehrerausbildung. München: kopaed.

Brandhofer, Gerhard, Angela Kohl, Marlene Miglbauer, und Thomas Nárosy. 2016. «digi.kompP - Digitale Kompetenzen für Lehrende Das digi.kompP-Modell im internationalen Vergleich und in der Praxis der österreichischen Pädagoginnen- und Pädagogenbildung». Open Online Journal for Research and Education 6: 38-51. https://journal.ph-noe.ac.at/index.php/ resource/article/view/305.

Cress, Ulrike, Ira Diethelm, Birgit Eickelmann, Olaf Köller, Reinhold Nickolaus, Hans Anand Pant, und Kristina Reiss. 2018. Schule in der digitalen Transformation - Perspektiven der Bildungswissenschaften (acatech DISKUSSION), München. https://www.acatech.de/Publikation/schule-in-der-digitalentransformation-perspektiven-der-bildungswissenschaften.

Eickelmann, Birgit, Ramona Lorenz, und Manuela Endberg. «Die Relevanz der Phasen der Lehrerausbildung hinsichtlich der Vermittlung didaktischer und methodischer Kompetenzen für den schulischen Einsatz digitaler Medien in Deutschland und im Bundesländervergleich». In Schule digital - der Länderindikator 2016. Kompetenzen von Lehrpersonen der Sekundarstufe I im Umgang mit digitalen Medien im Bundesländervergleich, hrsg. v. Wilfried Bos, Ramona Lorenz, Manuela Endberg, Birgit Eickelmann, Rudolf Kammerl, und Stefan Welling, 148-179. Münster: Waxmann. https://www.waxmann.com/buch3540.

Endberg, Manuela. 2019. Professionswissen von Lehrpersonen zum Einsatz digitaler Medien im Unterricht. Eine explorative empirische Untersuchung mit einer repräsentativen Stichprobe von Lehrpersonen der Sekundarstufe I in Deutschland. Münster: Waxmann.

Endberg, Manuela, und Ramona Lorenz. 2016. «Selbsteinschätzung medienbezogener Kompetenzen von Lehrkräften in Deutschland und im Bundesländervergleich». In Schule digital - der Länderindikator 2016. Kompetenzen von Lehrpersonen der Sekundarstufe I im Umgang mit digitalen Medien im Bundesländervergleich, hrsg. v. Wilfried Bos, Ramona Lorenz, Manuela Endberg, Birgit Eickelmann, Rudolf Kammerl, und Stefan Welling, 180-208. Münster: Waxmann. https://www.waxmann.com/buch3540. 
Endberg, Manuela, und Ramona Lorenz. 2017. «Medienbezogene Kompetenzen von Lehrkräften in der Sekundarstufe I im Bundesländervergleich und im Trend von 2016 bis 2017». In Schule digital - der Länderindikator 2017. Schulische Medienbildung mit besonderem Fokus auf MINT-Fächer in der Sekundarstufe I im Bundesländervergleich und Trends von 2015 bis 2017, hrsg. v. Ramona Lorenz, Wilfried Bos, Manuela Endberg, Birgit Eickelmann, Silke Grafe, und Jan Vahrenhold, 151-177. Münster: Waxmann. https://www.waxmann.com/ buch3699.

Endberg, Manuela, Nicole Rolf, und Ramona Lorenz. 2018. Schule digital - Handreichung zur schulischen Medienarbeit. Münster: Waxmann.

Ferrari, Anusca. 2013. DigComp: A Framework for Developing and Understanding Digital Competence in Europe, Seville: JRC-IPTS. Zugriff 09.01.2019. http://ipts.jrc.ec.europa.eu/publications/pub.cfm?id=6359.

Ghomi, Mina und Redecker, Christine. 2019. «Digital Competence of Educators (DigCompEdu): Development and Evaluation of a Self-Assessment Instrument for Teachers) Digital Competence». CSEDU 2019 - Proceedings of the 11th International Conference on Computer Supported Education. https://doi.org/10.5220/0007679005410548.

Graham, Charles R. 2011. "Theoretical considerations for understanding technological pedagogical content knowledge (TPACK)». Computers \& Education 57 (3): 1953-1960. https:// doi.org/10.1016/j.compedu.2011.04.010.

Herring, Mary C., Matthew J. Koehler, und Punya Mishra, Hrsg. 2016. Handbook of Technological Pedagogical Content Knowledge (TPACK) for Educators. Second Edition. New York: Routledge.

Herzig, Bardo. 2004. «Medienpädagogische Kompetenz». In Handbuch Lehrerbildung, hrsg. v. Sigrid Blömeke, Peter Reinhold, Gerhard Tulodziecki, und Johannes Wild, 578-594. Bad Heilbrunn: Klinkhardt.

Herzig, Bardo, und Alexander Martin. 2018. «Lehrerbildung in der digitalen Welt - konzeptionelle und empirische Aspekte». In Digitalisierung und Bildung, hrsg. v. Julia Knopf, Silke Ladel, und Armin Weinberger, 89-113. Wiesbaden: Springer VS Verlag.

Herzig, Bardo, Alexander Martin, Niclas Schaper, und Daniel Ossenschmidt. 2015. «Modellierung und Messung medienpädagogischer Kompetenz - Grundlagen und erste Ergebnisse». In Kompetenzerwerb an Hochschulen: Modellierung und Messung. Zur Professionalisierung angehender Lehrerinnen und Lehrer sowie frühpädagogischer Fachkräfte, hrsg. v. Barbara Koch-Priewe, Anna Köker, Jürgen Seifried, und Eveline Wuttke, 153-176. Bad Heilbrunn: Julius Klinkhardt.

Kimmons, Royce 2015. «Examining TPACK's theoretical future». Journal of Technology and Teacher Education 23 (1): 53-77. https://www.learntechlib.org/primary/p/130072/.

KMK [Sekretariat der Ständigen Konferenz der Kultusminister der Länder in der Bundesrepublik Deutschland]. 1998. "Zur Rolle der Medienpädagogik,insbesondere der Neuen Medien und der Telekommunikation in der Lehrerbildung - Bericht des Schulausschusses vom 11.12.1998». Zugriff 09.01.2019. https://www.kmk.org/fileadmin/Dateien/pdf/PresseUndAktuelles/Beschluesse_Veroeffentlichungen/neuemed.pdf. 
KMK [Sekretariat der Ständigen Konferenz der Kultusminister der Länder in der Bundesrepublik Deutschland]. 2014. "Standards für die Lehrerbildung: Bildungswissenschaften (Beschluss der Kultusministerkonferenz vom 16.12.2004 i. d. F. vom 12.06.2014)». Zugriff 09.01.2019. https://www.kmk.org/fileadmin/Dateien/veroeffentlichungen_ beschluesse/2004/2004_12_16-Standards-Lehrerbildung-Bildungswissenschaften.pdf.

KMK [Sekretariat der Ständigen Konferenz der Kultusminister der Länder in der Bundesrepublik Deutschland]. 2016. «Bildung in der digitalen Welt. Strategie der Kultusministerkonferenz». Zugriff 09.01.2019. https://www.kmk.org/fileadmin/Dateien/pdf/PresseUndAktuelles/2018/Digitalstrategie_2017_mit_Weiterbildung.pdf.

Koehler, Matthew J., und Punya Mishra. 2009. "What Is Technological Pedagogical Content Knowledge?» Contemporary Issues in Technology and Teacher Education 9 (1): 60-70.

Koehler, Matthew J., Punya Mishra, Kristen Kereluik, Tae Seob Shin, und Charles R. Graham. 2014. "The technological pedagogical content knowledge framework». In Handbook of research on educational communications and technology, hrsg. v. J. Michael Spector, M. David Merrill, Jan Elen, und M. J. Bishop, 101-111. New York: Springer Science+Business Media. https://www.learntechlib.org/primary/p/29544/.

Kushner Benson, Susan N. Cheryl, I. Ward, und Xin Liang. 2015. «The essential role of pedagogical knowledge in technology integration for transformative teaching and learning». In Technological Pedagogical Content Knowledge. Exploring, Developing, and Assessing TPCK, hrsg. v. Charoula Angeli und Nicos Valanides, 3-18, New York: Springer Science+Business Media.

Mishra, Punya, und Matthew J. Koehler. 2006. «Technological Pedagogical Content Knowledge: A Framework for Teacher Knowledge». Teachers College Record 108 (6): 1017-1054. https:// doi.org/10.1111/j.1467-9620.2006.00684.x.

Mouza, Chrystalla. 2016. «Developing and assessing TPACK among pre-service teachers. A synthesis of research». In Handbook of Technological Pedagogical Content Knowledge (TPACK) for Educators. Second Edition, hrsg. v. Mary C. Herring, Matthew J. Koehler, und Punya Mishra, 169-190, New York: Routledge.

Netzwerk Digitale Bildung. 2018. Wegweiser digitale Bildung. Für zeitgemäßen Unterricht mit digitalen Medien. Zugriff 09.01.2019. https://www.netzwerk-digitale-bildung.de.

Niess, Margaret Louise. 2013. «Central Component Descriptors for Levels of Technological Pedagogical Content Knowledge». Journal of Educational Computing Research 48 (2): 173-198. https://doi.org/10.2190/EC.48.2.d.

Niess, Margaret Louise, und Henry Gillow-Wiles. 2017. «Expanding teachers' technological pedagogical reasoning with a systems pedagogical approach». Australasian Journal of Educational Technology 33 (3): 77-95. https://doi.org/10.14742/ajet.3473.

Redecker, Christine. 2017. European Framework for the Digital Competence of Educators. DigCompEdu. https://doi.org/10.2760/159770. 
Schiefner-Rohs, Mandy. 2018. «Medienbildung in der Lehrer*innenbildung an der Hochschule: Über Bricolage zur Reflexion». In Schule 4.0. Zukunftstrends, Rahmenbedingungen, Praxisbeispiele, hrsg. v. Katharina Scheiter, und Thomas Riecke-Baulecke, 56-68. München: Cornelsen.

Shulman, Lee S. 1986. «Those Who Understand: Knowledge Growth in Teaching». Educational Researcher 15 (2): 4-14. https://doi.org/10.3102/0013189X015002004.

Shulman, Lee S. 1987. "Knowledge and Teaching: Foundations of the New Reform». Harvard Educational Review 57 (1): 1-23. https://doi.org/10.17763/haer.57.1.j463w79r56455411.

Søby, Morten. 2003. Digital competence: from ICT skills to digital ,Bildung'. University of Oslo: ITU.

Tulodziecki, Gerhard. 2012. «Medienpädagogische Kompetenz und Standards in der Lehrerbildung». In Jahrbuch Medienpädagogik 9, hrsg. v. Renate Schulz-Zander, Birgit Eickelmann, Heinz Moser, Horst Niesyto, und Petra Grell, 271-297. Wiesbaden: VS Verlag für Sozialwissenschaften.

Valtonen, Teemu, Erkko Sointu, Kati Mäkitalo-Siegl, und Jari Kukkonen. 2015. «Developing a TPACK measurement instrument for 21st century pre-service teachers». Seminar.net - International journal of media, technology and lifelong learning 11 (2): 87-100. https://journals. hioa.no/index.php/seminar/article/view/2353.

Voogt, Joke, Petra Fisser, Natalie Pareja Roblin, Jo Tondeur, und Johan van Braak. 2013. «Technological pedagogical content knowledge - a review of the literature». Journal of Computer Assisted Learning 29 (2): 109-121. https://doi.org/10.1111/j.1365-2729.2012.00487.x.

Willermark, Sara. 2017. «Technological Pedagogical and Content Knowledge: A Review of Empirical Studies Published from 2011 to 2016». Journal of Educational Computing Research 56 (3): 315-343. https://doi.org/10.1177/0735633117713114. 